\title{
Successful Pregnancy Outcome after Fertility-sparing Surgery in a Woman with Invasive Epithelial Ovarian Carcinoma
}

\author{
${ }^{1}$ Nikita R Vijay, ${ }^{2}$ Anjali Kawthalkar
}

\begin{abstract}
Occurrence of epithelial ovarian carcinoma in young women is rare. Fertility-sparing surgery is a viable option for such women. We are reporting a case of a 24-year-old nulligravida woman, diagnosed with an ovarian lesion suspicious of malignancy, in whom unilateral salpingo-oophorectomy along with omentectomy and peritoneal biopsy was done. Histopathological report showed well-differentiated epithelial ovarian carcinoma [International Federation of Gynecology and Obstetrics (FIGO) stage IA, grade I], and after $1 \frac{1}{2}$ years of surgery, she conceived and delivered a healthy baby at term.
\end{abstract}

Keywords: Epithelial ovarian carcinoma, Fertility-sparing surgery, Unilateral salpingo-oophorectomy.

How to cite this article: Vijay NR, Kawthalkar A. Successful Pregnancy Outcome after Fertility-sparing Surgery in a Woman with Invasive Epithelial Ovarian Carcinoma. J South Asian Feder Obst Gynae 2018;10(1):61-62.

Source of support: Nil

Conflict of interest: None

Date of received: 15 January 2018

Date of acceptance: 25 February 2018

Date of publication: May 2018

\section{INTRODUCTION}

Ovarian cancer is the deadliest of all malignancies of the female genital tract. Ovarian cancer is predominantly a disease of older, postmenopausal women. Majority of cases (90\%) are of epithelial origin. About 7 to $8 \%$ of epithelial ovarian cancers occur among women $<35$ years of age. ${ }^{1}$ Standard surgical treatment for epithelial ovarian cancer is staging laparotomy with total hysterectomy with bilateral salpingo-oophorectomy. Such type of management leads to permanent infertility. Young patients with

\footnotetext{
${ }^{1}$ Assistant Professor, ${ }^{2}$ Professor

1,2Department of Obstetrics and Gynecology, NKP Salve Institute of Medical Sciences \& Research Center and Lata Mangeshkar Hospital, Nagpur, Maharashtra, India

Corresponding Author: Nikita R Vijay, Assistant Professor Department of Obstetrics and Gynecology, NKP Salve Institute of Medical Sciences \& Research Center and Lata Mangeshkar Hospital, Nagpur, Maharashtra, India, Phone: +917104306171 e-mail: nikitarvijay@gmail.com
}

strong desire for fertility need conservative approach called fertility-sparing surgery, surgery that preserves reproductive potential without compromising curability. However, this conservative management in the form of unilateral cystectomy, unilateral salpingo-oophorectomy, or bilateral cystectomy is done if tumor is bilateral; also omentectomy, peritoneal sampling, and lymph node sampling have been approved by various international and national societies and need careful evaluation. Evaluation includes FIGO stage, histology, grade of tumor, and cancer antigen 125 (CA-125) value. Some of the patients need postoperative chemotherapy also.

\section{CASE REPORT}

A 24-year-old, nulligravida woman, married since 3 years, reported with history of pain in abdomen and irregular menses. Ultrasonography of abdomen (pelvis) revealed normal-sized uterus with normal endomyometrial complex. Thick-walled cystic mass of $6 \times 5 \times 5$ $\mathrm{cm}$ in right ovary with approximate volume of $72 \mathrm{~cm}^{3}$ with multiple mural polypoidal echogenic nodules was observed. Neoplastic right ovary, left ovary normal (3 $\times 2 \times 1.5 \mathrm{~cm}$ ), no free fluid in pouch of douglas were found. Serum CA-125 value was $127 \mathrm{mIU} / \mathrm{mL}$. She was posted for staging laparotomy after proper counseling with informed risk of possibility of malignancy, need for postoperative chemotherapy, including need for assisted reproductive techniques (ARTs), or ovum donation in future. Intraoperative findings included normal uterus, right ovary $6 \times 6 \mathrm{~cm}$ in size, intact capsule with smooth surface, normal-appearing left ovary, and no ascitis. Cut section showed solid areas with papillary projections. Frozen section examination could not be done, as the facility was not available. Histopathological report revealed well-differentiated serous papillary cyst adenocarcinoma with unremarkable omental and peritoneal biopsy (FIGO stage IA, grade I), and she came for follow-up in 6 months and then was lost to follow-up.

She again reported at 9 months of her pregnancy for antenatal checkup. She had consulted a private practitioner, first time at her 4th month of pregnancy. Ultrasonography of abdomen and CA-125 were advised during her 7th month, and reports were normal. Emergency 
lower segment cesarean section (LSCS) was done at 38 weeks in view of fetal distress. The LSCS was uneventful and the opposite ovary was grossly normal. Postoperative serum CA- 125 was repeated and was normal. She was advised to undergo hysterectomy with left-sided salpingo-oophorectomy 6 months after LSCS.

\section{DISCUSSION}

Fertility-sparing surgery for reproductive-age patients with invasive epithelial ovarian carcinoma has been adopted for stage IA and nonclear cell histology grade I (G1)/grade 2 (G2) according to the 2007 guidelines of the American College of Obstetrics and Gynecology (ACOG) and for unilateral stage I tumor without dense adhesions showing favorable histology (i.e., nonclear cell histology G1/2) according to the 2008 guidelines of the European Society for Medical Oncology. In Japan, fertility-sparing surgery has been recommended for patients with stage IA tumor or unilateral stage IC tumor based on intraoperable capsule rupture IC(b) and favorable histology, according to the 2004 guidelines and the 2007 guidelines of the Japan Society of Gynecology Oncology. Platinumbased chemotherapy is advised in women with high-risk cancer (FIGO stage IA, G2, or higher) as well as in clear cell carcinoma after fertility-sparing surgery. ${ }^{2}$

At present, more than 220 pregnancies after ovarian cancer have been reported, with an overall miscarriage rate of $17 \%$. Overall 5 -year survival rates have been reported to be 87 to $90 \%$ with approximately $12 \%$ of patients showing cancer recurrence after fertility-sparing surgery. Laparoscopic fertility-sparing surgery is also an approach in FIGO stage IA, where 3-year survival is about $95 \%{ }^{3}$

The advent of ART has an impact on such type of management for early-stage ovarian cancer. ${ }^{4,5}$

\section{CONCLUSION}

Fertility-conservative surgery in early-stage epithelial ovarian cancer is a viable option for a young woman desirous of pregnancy. Spontaneous successful pregnancy after this surgery makes it a meaningful alternative to those young nulliparous women suffering from invasive epithelial ovarian cancer.

\section{REFERENCES}

1. Nam JH, Park JY. Fertility-sparing surgery for young women with early-stage epithelial ovarian cancer. Gynecol Obstet Invest 2013 Aug;76(1):14-24.

2. Fakhr I, Abd-Allah M, Ramzy S, Mohamed AM, Saber A. Outcome of fertility preserving surgery in early stage ovarian cancer. J Egypt Natl Canc Inst 2013 Dec;25(4):219-222.

3. Feichtinger M, Rodriguez-Wallberg KA. Fertility preservation in women with cervical, endometrial or ovarian cancers. Gynecol Oncol Res Pract 2016 Jul;3:8.

4. Fotopoulou C, Braicu I, Sehouli J. Fertility-sparing surgery in early epithelial ovarian cancer: a viable option? Obstet Gynecol Int 2012 Dec;2012:238061.

5. Ditto A, Martinelli F, Lorusso D, Haeusler E, Carcangiu M, Raspagliesi F. Fertility sparing surgery in early stage epithelial ovarian cancer. J Gynecol Oncol 2014 Oct;25(4):320-327. 